\title{
ON DUAL BAER MODULES
}

\author{
DERYA KESKIN TÜTÜNCÜ \\ Department of Mathematics, Hacettepe University, 06800 Beytepe, Ankara, Turkey \\ e-mail:keskin@hacettepe.edu.tr \\ and RACHID TRIBAK \\ Département de Mathématiques, Faculté des Sciences de Tétouan, B.P 21.21. Tétouan, Morocco \\ e-mail:tribak12@yahoo.com
}

(Received 6 December 2008; revised 20 April 2009; accepted 25 August 2009; first published online 25 November 2009)

\begin{abstract}
In this paper we introduce $\mathcal{T}$-non-cosingular modules, dual Baer modules and $\mathcal{K}$-modules. We prove that a module $M$ is lifting and $\mathcal{T}$-non-cosingular if and only if it is a dual Baer and $\mathcal{K}$-module. Rings for which all modules are dual Baer are precisely determined. We also give a necessary condition for a finite direct sum of dual Baer modules to be dual Baer.
\end{abstract}

2000 Mathematics Subject Classification. 16D10, 16D80, 16 E60.

1. Introduction. Throughout this paper $S$ will denote the endomorphism ring of any module $M$. In [11] and [12], the authors investigate Baer modules and $\mathcal{K}$ non-singular modules. Motivated by these works, we introduce dual notions, these of dual Baer modules and $\mathcal{T}$-non-cosingular modules. A module $M$ is called a dual Baer module if for every $N \leq M$, there exists an idempotent $e$ in $S$ such that $\mathrm{D}(N)=\{\varphi \in$ $S \mid \operatorname{Im} \varphi \subseteq N\}=e S$. The module $\mathbb{Z}_{\mathbb{Z}}$ is not dual Baer, because for every integer $n \geq 2$, $\mathrm{D}(n \mathbb{Z})$ is a non-zero and proper right ideal of $\operatorname{End}(\mathbb{Z})$. On the other hand, the modules $\mathbb{Q}_{\mathbb{Z}}$ and $\mathbb{Z}\left(p^{\infty}\right)$ are dual Baer for every prime $p$ (see Corollary 2.4). A module $M$ is called a $\mathcal{T}$-non-cosingular module if, for every non-zero endomorphism $\varphi$ of $M, \operatorname{Im} \varphi$ is not small in $M$. Following [14], the module $M$ is called non-cosingular if for every non-zero module $N$ and every non-zero homomorphism $f: M \rightarrow N, \operatorname{Im} f$ is not a small submodule of $N$. It is clear that every non-cosingular module is $\mathcal{T}$-non-cosingular.

A module $M$ is called a lifting module if for every submodule $N$ of $M$, there is a decomposition $M=M_{1} \oplus M_{2}$ such that $M_{1} \leq N$ and $N \cap M_{2} \ll M_{2}$, or equivalently, for every submodule $N$ of $M$ there is a direct summand $K$ of $M$ such that $N / K \ll M / K$.

A ring $R$ is called a right Harada ring if every injective right $R$-module is lifting (see [2, 28.1 and 28.10]).

The aim of this paper is to study dual Baer modules. $\mathcal{T}$-non-cosingular modules will be studied in a subsequent paper.

Section 2 is devoted to the study of dual Baer modules. We will begin by providing an equivalent formulation of dual Baer modules (Theorem 2.1). Then we show that $R_{R}$ is dual Baer if and only if the ring $R$ is semi-simple. We also prove Theorem 2.14 which 
exhibits the connections between dual Baer modules and lifting modules. Moreover, we characterize right hereditary right Harada rings in terms of lifting dual Baer modules (Proposition 2.15).

In Section 3 we will be concerned with the direct sums of dual Baer modules. The structure of dual Baer modules over Dedekind rings is described explicitly.

2. Dual Baer modules. Rizvi and Roman introduced the concept of Baer modules in [11]. Let $M$ be a module. According to [11], $M$ is called a Baer module if for all $N \leq M$, the left annihilator of $N$ in $S, l_{S}(N)=S e$, with $e^{2}=e \in S$. Let $N \leq M$. In this paper we introduce the right ideal $\mathrm{D}(N)=\{\varphi \in S \mid \operatorname{Im} \varphi \subseteq N\}$ of $S$ as the dual notion of left annihilator $l_{S}(N)$ of $N$ in $S$. Clearly, $\mathrm{D}(e(M))=e S$ for any idempotent $e$ in $S$. A module $M$ is called dual Baer if for every $N \leq M$, there exists an idempotent $e$ in $S$ such that $\mathrm{D}(N)=e S$. It is obvious that any module with semi-simple endomorphism ring is dual Baer. The module $M$ is said to have the (strong) summand sum property, denoted briefly by (SSSP) SSP, if the sum of (any family of) two direct summands of $M$ is a direct summand of $M$. Next, we provide a characterization of dual Baer modules in terms of SSSP.

THEOREM 2.1. The following are equivalent for a module $M$ :

(i) $M$ is dual Baer.

(ii) For every subset $A$ of $S, \sum_{f \in A} \operatorname{Imf}=e(M)$ where $e=e^{2} \in S$.

(iii) For every right ideal I of $S, \sum_{f \in I} \operatorname{Imf}=e(M)$ where $e=e^{2} \in S$.

(iv) $M$ has the SSSP and for every $\varphi: M \rightarrow M, \operatorname{Im} \varphi$ is a direct summand of $M$.

Proof. (i) $\Rightarrow$ (ii) Let $A \subseteq S$. Let $N=\sum_{f \in A} \operatorname{Im} f \leq M$. Since $M$ is dual Baer, there exists an idempotent $e \in S$ such that $\mathrm{D}(N)=e S$. Thus $e(M) \subseteq N$. On the other hand, for every $f \in A$, we have $f \in \mathrm{D}(N)=e S$. Therefore for every $f \in A$, there exists $s \in S$ such that $f=e s$. It follows that for every $f \in A, \operatorname{Im} f \subseteq e(M)$. This gives that $N \subseteq e(M)$. Consequently, $N=e(M)$.

(ii) $\Rightarrow$ (iv) It is a consequence of the fact that every direct summand of $M$ is an epimorphic image of $M$.

(iv) $\Rightarrow$ (iii) Clear.

(iii) $\Rightarrow$ (i) Let $N$ be a submodule of $M$. Consider the right ideal $I=\mathrm{D}(N)=\{f \in$ $S \mid \operatorname{Im} f \subseteq N\}$ of $S$. By hypothesis, $\sum_{f \in I} \operatorname{Im} f=e(M)$ for some $e=e^{2} \in S$. Then $e \in$ $\mathrm{D}(N)$ and hence $e S \subseteq \mathrm{D}(N)$. Now if $f \in \mathrm{D}(N)=I$, then $\operatorname{Im} f \subseteq e(M)$. Moreover, since $S=e S \oplus(1-e) S$, we get $f=e s_{1}+(1-e) s_{2}$ for some $s_{1}, s_{2} \in S$. Therefore $f=e s_{1}$ because $\operatorname{Im} f \subseteq e(M)$. Hence $f \in e S$. So $e S=\mathrm{D}(N)$. This completes the proof.

From Theorem 2.1 it follows easily that all semi-simple modules are dual Baer.

Note that if $R$ is a commutative ring, then for any dual Baer module $M$ and any $r \in R, M r$ is a direct summand of $M$.

Corollary 2.2. A module $M$ is an indecomposable dual Baer module if and only if for every non-zero $\varphi \in S$, $\varphi$ is an epimorphism.

Proof. By Theorem 2.1. 
In view of the above corollary, every indecomposable dual Baer module $M$ is cohopfian (i.e. every monomorphism from $M$ to $M$ is an isomorphism). Let $R$ be a commutative Noetherian local complete domain with maximal ideal $m$. Then $E(R / m)$ is dual Baer by [13, page 143, Corollary 2] and Corollary 2.2.

Corollary 2.3. Every dual Baer module $M$ is $\mathcal{T}$-non-cosingular.

Proof. Let $M$ be a dual Baer module and let $\varphi \in \operatorname{End}(M)$ with $\operatorname{Im} \varphi \ll M$. By Theorem 2.1, $\operatorname{Im} \varphi$ is a direct summand of $M$. Therefore $\operatorname{Im} \varphi=0$, and $\operatorname{so} \varphi=0$.

COROLlary 2.4. Let $M$ be an injective $R$-module over a right hereditary ring $R$. Then $M$ is dual Baer if and only if $M$ has the SSSP. In particular, every indecomposable injective R-module is dual Baer.

Proof. Let $f$ be an endomorphism of $M$. Since $\operatorname{Im} f$ is a factor module of $M$ and $R$ is right hereditary, $\operatorname{Im} f$ is injective. Hence $\operatorname{Im} f$ is a direct summand of $M$. The result follows from Theorem 2.1.

Corollary 2.5. Let $M$ be a dual Baer module. Then every direct summand of $M$ is also dual Baer.

Proof. Let $M=N \oplus N^{\prime}$. Since $M$ has the SSSP, it is easy to see that $N$ has the SSSP. Now let $f: N \rightarrow N$ be any endomorphism of $N$. Consider the homomorphism $f \oplus 0_{N^{\prime}}$ : $N \oplus N^{\prime} \rightarrow N \oplus N^{\prime}$ defined by $f \oplus 0_{N^{\prime}}\left(n+n^{\prime}\right)=f(n)$. Now $f \oplus 0_{N^{\prime}}\left(N \oplus N^{\prime}\right)=f(N)$ is a direct summand of $M$ and hence it is a direct summand of $N$. Therefore $N$ is dual Baer by Theorem 2.1.

Corollary 2.6. (i) Every dual Baer module is a direct sum of indecomposable modules.

(ii) Every dual Baer lifting module is a direct sum of hollow modules.

Proof. (i) By Theorem 2.1 and [10, Theorem 2.17].

(ii) By (i) and [2, 22.2 and 22.6].

A module $M$ is called a regular module if every cyclic submodule of $M$ is a direct summand of $M$ (see [8, Page 272, Exercise 16]).

COROLlaRY 2.7. If $M$ is a regular dual Baer module, then $M$ is semi-simple.

Proof. Let $N \leq M$. Note that $N=\sum_{x \in N} x R$. By Theorem 2.1, $N$ is a direct summand of $M$.

PROPOSITION 2.8. If $R_{R}$ is dual Baer, then the ring $R$ is von Neumann regular.

Proof. Take any principal right ideal $I=a R$ of $R$. Consider the $R$-homomorphism $f: R_{R} \longrightarrow R_{R}$ defined by $f(r)=a r$, where $r \in R$. Then $\operatorname{Im} f=I$, which is a direct summand of $R_{R}$ by hypothesis. So $R$ is von Neumann regular. 
COROLlary 2.9. Let $R$ be a ring. Then the following are equivalent:

(i) $R_{R}$ is dual Baer.

(ii) $R_{R}$ is semi-simple.

(iii) ${ }_{R} R$ is dual Baer.

(iv) ${ }_{R} R$ is semi-simple.

Proof. (i) $\Leftrightarrow$ (ii) Assume $R_{R}$ is dual Baer. By Proposition 2.8, $R$ is von Neumann regular. Therefore $R_{R}$ is semi-simple by Corollary 2.7. The converse is clear.

(iii) $\Leftrightarrow$ (iv) The proof runs as before.

(ii) $\Leftrightarrow$ (iv) By [8, Theorem 8.2.1].

COROLlARY 2.10. The following are equivalent for any ring $R$ :

(i) Every right $R$-module is dual Baer.

(ii) Every left $R$-module is dual Baer.

(ii) $R$ is semi-simple.

LEMma 2.11. Let $N$ be a submodule of a $\mathcal{T}$-non-cosingular module $M$ and let e be an idempotent in $S$. If $e(M) \leq N$ and $N / e(M) \ll M / e(M)$, then $\mathrm{D}(N)=e S$.

Proof. Since $e(M) \leq N$, we have $e S \subseteq \mathrm{D}(N)$. Now let $\varphi \in \mathrm{D}(N)$ and let us prove that $\varphi \in e S$. Note that $M=e(M) \oplus(1-e)(M)$ and $N / e(M) \ll M / e(M)$. Therefore $N \cap(1-e)(M) \ll M$. Since $S=e S \oplus(1-e) S$, there exist $s_{1}$ and $s_{2}$ in $S$ such that $\varphi=e s_{1}+(1-e) s_{2}$. Thus $\operatorname{Im}(1-e) s_{2} \leq N \cap(1-e)(M) \ll M$. By hypothesis, we get $(1-e) s_{2}=0$ and hence $\varphi=e s_{1} \in e S$.

A module $M$ is called a $\mathcal{K}$-module if, for every non-small submodule $N$ of $M$, there exists a non-zero endomorphism $\varphi$ of $M$ such that $\varphi^{-1}(N)=M$. This is obviously equivalent to the condition that, $\mathrm{D}(N) \neq 0$ for every non-small submodule $N$ of $M$. Note that $\mathbb{Q}_{\mathbb{Z}}$ is not a $\mathcal{K}$-module since it contains a proper non-small submodule and every non-zero endomorphism of $\mathbb{Q}_{\mathbb{Z}}$ is an isomorphism. On the other hand, $\mathbb{Z}$ is a $\mathcal{K}$-module since every non-zero submodule of $\mathbb{Z}$ is isomorphic to $\mathbb{Z}$.

LEMMA 2.12. Every lifting module $M$ is a $\mathcal{K}$-module.

Proof. Let $N \leq M$ such that $\mathrm{D}(N)=0$. By the lifting property, there exists a direct summand $K$ of $M$ such that $N / K \ll M / K$. Now there exists an idempotent $e \in S$ such that $e(M)=K$. This implies that $e \in \mathrm{D}(N)$. By hypothesis, $e=0$. Therefore $K=0$ and hence $N \ll M$.

Proposition 2.13. Let $M$ be a dual Baer $\mathcal{K}$-module. Then $M$ is lifting.

Proof. Let $N$ be any non-small submodule of $M$. Then $\mathrm{D}(N)=e S$ for some non-zero idempotent $e \in S$. Thus Ime is a non-zero direct summand of $M$ which is contained in $N$. Moreover, if $f$ is another idempotent in $S$ such that $\operatorname{Im} e \leq \operatorname{Im} f \leq N$, then $f \in \mathrm{D}(N)$. Therefore there exists $s \in S$ such that $f=e s$. So $\operatorname{Im} f \leq \operatorname{Im} e$ and hence $\operatorname{Im} e=\operatorname{Im} f$. This proves that Ime is a maximal direct summand of $M$ with $\operatorname{Im} e \leq N$. By $[\mathbf{1 5}, 41.12], M$ is lifting. 
THEOREM 2.14. The following statements are equivalent for a module $M$ :

(i) $M$ is a lifting $\mathcal{T}$-non-cosingular module.

(ii) $M$ is a dual Baer and $\mathcal{K}$-module.

Proof. By Lemmas 2.11, 2.12, Corollary 2.3 and Proposition 2.13.

Recall that a module $M$ is uniserial if its submodules are linearly ordered by inclusion and it is serial if it is a direct sum of uniserial submodules. The ring $R$ is right (left) serial if the right (left) $R$-module $R_{R}\left({ }_{R} R\right)$ is serial and it is serial if it is both right and left serial.

Theorem 2.14 is a useful source of examples of dual Baer modules. In fact, since every non-cosingular module is $\mathcal{T}$-non-cosingular, every non-cosingular lifting module is dual Baer. By using this fact, we will construct the following examples.

(1) If $R$ is a right hereditary ring, then every injective module is non-cosingular by [14, Proposition 2.7]. Thus every injective lifting module is dual Baer.

(2) If $R$ is a right Harada ring, then every injective module is lifting. Therefore every injective non-cosingular module is dual Baer.

(3) If the ring $R$ is artinian serial with $(\operatorname{Rad}(R))^{2}=0$, then every module is lifting by $[2,29.10]$. So every non-cosingular module is dual Baer.

PROPOSITION 2.15. The following statements are equivalent for a ring $R$ :

(i) $R$ is a right hereditary right Harada ring.

(ii) Every injective module is lifting dual Baer.

Proof. (i) $\Rightarrow$ (ii) Let $M$ be an injective module. It is clear that $M$ is lifting. By [5, Proposition 1.6], $M$ has the SSP. Thus $M$ has the SSSP by [4, Proposition 4.9]. Therefore $M$ is dual Baer by Corollary 2.4.

(ii) $\Rightarrow$ (i) It follows from [5, Proposition 1.6].

What is lacking is an explicit example. Let $k$ be a field and let $R$ be the ring of $n \times n$ upper triangular matrices over $k$. By [9, Example 2.36], $R$ is an artinian right hereditary ring and $\operatorname{Rad}(R)$ consists of all matrices in $R$ with a zero diagonal. Thus $(\operatorname{Rad}(R))^{2}=0$. On the other hand, $R$ is a serial ring by [3, Example 1.21]. It follows that every injective $R$-module is dual Baer.

Theorem 2.14 and Proposition 2.15 show the importance of dual Baer modules in the theory of lifting modules, hereditary rings, and Harada rings.

In [14], the authors defined $\bar{Z}(M)=\cap\{\operatorname{Ker}(g): g \in \operatorname{Hom}(M, N), N \ll E(N)\}$ and $\bar{Z}^{2}(M)=\bar{Z}(\bar{Z}(M))$, where $E(N)$ is the injective hull of $N$.

Proposition 2.16. Let $M$ be a lifting module. Then $\bar{Z}^{2}(M)$ is a direct summand of $M$ which is dual Baer.

Proof. By [14, Corollary 3.4 and Theorem 4.1] and Theorem 2.14.

Proposition 2.17. Let $M$ be an indecomposable dual Baer module with finite uniform dimension. Then $S$ is semi-local. 
Proof. By Corollary 2.2 and [1, Theorem 5].

Note that the ring of endomorphisms of a dual Baer module may not be von Neumann regular. Also if the ring of endomorphisms of any module $M$ is von Neumann regular, then $M$ need not be dual Baer.

EXAMPLE 2.18. ( $i)$ Let $M$ be the Prüfer p-group $\mathbb{Z}\left(p^{\infty}\right)$. It is dual Baer by Corollary 2.4. But $S=\operatorname{End}(M)$ is not von Neumann regular.

(ii) Let $K$ be a field and let $R=\prod_{i=1}^{\infty} K_{i}$ with $K_{i}=K$ for $i=1,2, \ldots$ In [8, Page $264]$, it is proven that the ring $R$ is von Neumann regular which is not semi-simple. Thus $R_{R}$ is not dual Baer by Corollary 2.9. But End $(R)$ is von Neumann regular.

In this vein we can give the following result.

Proposition 2.19. (i) Let $M$ be a dual Baer module such that for every endomorphism $f$ of $M$, Kerf is a direct summand of $M$. Then $S$ is von Neumann regular.

(ii) Let $M$ be a module. If $S$ is von Neumann regular and $M$ has the SSSP, then $M$ is dual Baer.

Proof. By Theorem 2.1 and [8, Page 272, Exercise 17].

3. Direct sums of dual Baer modules. If $R$ is a Dedekind domain, then $R$ is said to be proper if $R$ is not a field. If $R$ is a proper Dedekind domain, then for each nonzero prime ideal $P$ of $R, R\left(P^{\infty}\right)$ will denote the $P$-primary component of the torsion $R$-module $K / R$, where $K$ is the quotient field of $R$. To prove Theorem 3.4 we need the following three results.

EXAMPLE 3.1. Let $R$ be a proper Dedekind domain. Let $P$ be any non-zero prime ideal of $R$. Consider the module $M=R\left(P^{\infty}\right) \oplus R / P$ and the endomorphism $f: M \longrightarrow M$ defined by $f(x+\bar{y})=$ cy with $x \in R\left(P^{\infty}\right), y \in R$ and $c$ is a non-zero element of $R\left(P^{\infty}\right)$ such that $c P=0$. It is clear that Imf $=c R$ which is non-zero and small in $M$. So $M$ is not a $\mathcal{T}$-non-cosingular module. In particular, for any prime integer $p$, the $\mathbb{Z}$-module $\mathbb{Z}\left(p^{\infty}\right) \oplus \mathbb{Z} / p \mathbb{Z}$ is not a $\mathcal{T}$-non-cosingular $\mathbb{Z}$-module.

LEMMA 3.2. Let $L=x R$ be a cyclic module over a commutative ring $R$. Then $L$ is dual Baer if and only if $L$ is semi-simple.

Proof. Let $y \in L$. Then there exists $r \in R$ such that $y=x r$. Consider the endomorphism $f$ of $L$ defined by $f(x \alpha)=y \alpha$. The map $f$ is well defined since $R$ is commutative. As $L$ is dual Baer, $y R$ is a direct summand of $L$ by Theorem 2.1. Applying Theorem 2.1 again, $L$ has SSSP, and hence every submodule of $L$ is a direct summand. Therefore $L$ is semi-simple. The converse is clear.

Lemma 3.3. Suppose that $R$ is a commutative ring which is not semi-simple. Let $M$ be an indecomposable module containing an element $x$ such that $x \notin \operatorname{Rad}(M)$ and $\operatorname{Ann}_{R}(x)=0$. Then $M$ is not dual Baer. 
Proof. Suppose that $M$ is dual Baer. Since $x \notin \operatorname{Rad}(M), x R$ is not small in $M$. Let $L$ be a proper submodule of $M$ such that $x R+L=M$. If $x R \cap L=0$, then $M=x R$ which is isomorphic to $R$ since $A n n_{R}(x)=0$. Thus $R$ is dual Baer and hence $R$ is semi-simple by Corollary 2.9. This contradicts our assumption. So $x R \cap L \neq 0$. Let $0 \neq r \in R$ such that $x r \in L$. Now consider the endomorphism of $M$ defined by $f(y)=y r$ for every $y \in M$. Since $f \neq 0, \operatorname{Im} f=M$ by Corollary 2.2. But $\operatorname{Im} f=M r=$ $(x r) R+L r \leq L$. Thus $L=M$, a contradiction. Consequently, $M$ is not dual Baer.

THEOREM 3.4. Let $R$ be a proper Dedekind domain with quotient field $K$. The following are equivalent for an $R$-module $M$ :

(i) $M$ is dual Baer.

(ii) $M$ is a direct sum of copies of $K,\left(R\left(P_{i}^{\infty}\right)\right)_{i \in I}$ and $\left(R / Q_{j}\right)_{j \in J}$ where $\left(P_{i}\right)_{i \in I}$ and $\left(Q_{j}\right)_{j \in J}$ are non-zero prime ideals of $R$ with $P_{i} \neq Q_{j}$ for every couple $(i, j) \in I \times J$.

Proof. (i) $\Rightarrow$ (ii) Since $M$ is dual Baer, $M=\oplus_{k \in \Lambda} M_{k}$ is a direct sum of indecomposable submodules $M_{k}(k \in \Lambda)$ by Corollary 2.6. By [7, Theorem 10], each $M_{k}$ is either isomorphic to $R\left(P^{\infty}\right)$ or $R / P^{n}$ for some prime ideal $P$ or $M_{k}$ is torsion-free. Note that if $M_{k}$ is isomorphic to $R / P^{n}$, then $P^{n}=P$ by Corollary 2.5 and Lemma 3.2. Now if $M_{k}$ is a non-divisible torsion-free module, then $M_{k}$ is isomorphic to $K$ (see [7, Theorem 7] and Lemma 3.3). The proof of the necessity is completed by Example 3.1 and Corollaries 2.3 and 2.5.

(ii) $\Rightarrow$ (i) It is well known that over $R$, a module $N$ is radical if and only if it is divisible if and only if it is injective. Let $M$ be a module having the structure described in the statement. By [7, Theorem 8], $M$ possesses a unique largest injective submodule $I(M)$. Note that $I(M)$ is the sum of all injective submodules of $M$. Moreover, $M=$ $I(M) \oplus S(M)$ where $S(M)=\left[\oplus_{j \in J} M_{Q_{j}}\right]$ is semi-simple and $M_{Q_{j}}$ is the $Q_{j}$-primary component of $T(M)$, the torsion submodule of $M$. Let $N$ and $L$ be submodules of $M$ such that $M=N \oplus L$. Then $N=I(N) \oplus N_{1}$ and $L=I(L) \oplus L_{1}$. Hence $M=$ $I(N) \oplus I(L) \oplus N_{1} \oplus L_{1}$. By [6, Lemma 2.1], $I(M)=I(N) \oplus I(K)$. So $N_{1} \oplus K_{1} \cong S(M)$ and hence $N_{1}$ is a direct summand of $S(M)$. Note that injective $R$-modules and semisimple $R$-modules all have the SSSP. Thus $I(M)$ and $S(M)$ have the SSSP. Therefore $M$ has the SSSP. On the other hand, if $f$ is an endomorphism of $M$, then $f(I(M))$ is injective since $R$ is an hereditary ring. So $f(I(M))$ is a direct summand of $I(M)$. This gives that $f(M)$ is a direct summand of $M$ since $f(S(M)$ ) is a direct summand of $S(M)$. Consequently, $M$ is dual Baer by Theorem 2.1.

Note that the last theorem gives many examples (see also Example 3.1) showing that a direct sum of dual Baer modules is not, in general, dual Baer.

Corollary 3.5. A $\mathbb{Z}$-module $M$ is dual Baer if and only if $M$ is isomorphic to a direct sum of arbitrarily many copies of $\mathbb{Q}$ and $\left(\mathbb{Z}\left(p_{i}^{\infty}\right)\right)_{i \in I}$ and $\left(\mathbb{Z} / q_{j} \mathbb{Z}\right)_{j \in J}$, where $p_{i}(i \in I)$ and $q_{j}(j \in J)$ are primes with $p_{i} \neq q_{j}$ for every couple $(i, j) \in I \times J$.

Proof. By Theorem 3.4.

THEOREM 3.6. Let $R$ be a non-local Dedekind domain. The following are equivalent for a module $M$ : 
(i) $M$ is dual Baer lifting.

(ii) $M$ is torsion and every P-primary component of $M$ is isomorphic either to $\left[R\left(P^{\infty}\right)\right]^{n_{P}}$ or $[R / P]^{\left(I_{P}\right)}$ for some natural number $n_{P}$ and index set $I_{P}$.

Proof. By Theorem 3.4, [10, Propositions A.7 and A.8].

COROLlary 3.7. A Z-module $M$ is dual Baer lifting if and only if $M$ is torsion and each p-primary component $M_{p}$ is isomorphic either to $\left[\mathbb{Z}\left(p^{\infty}\right)\right]^{n_{p}}$ or $[\mathbb{Z} / p \mathbb{Z}]^{\left(I_{p}\right)}$ for some natural number $n_{p}$ and index set $I_{p}$.

Proof. By Theorem 3.6.

Let $A$ and $B$ be modules. If for every homomorphism $\varphi: A \longrightarrow B, \operatorname{Im} \varphi$ is a direct summand of $B$, then we say that $A$ is relative $d$ to $B$. We call the modules $A$ and $B$ relatively $d$-modules if, $A$ is relative $d$ to $B$ and $B$ is relative $d$ to $A$.

Lemma 3.8. Let $M_{1}$ and $M_{2}$ be dual Baer relatively d-modules. Assume that $M_{2}$ is $M_{1}$-projective (or $M_{1}$ is $M_{2}$-projective). Then $M=M_{1} \oplus M_{2}$ is dual Baer.

Proof. Let $I=\left\{\varphi_{j} \mid j \in J\right\}$ be any subset of $S$. Let $K=\sum_{j \in J} \operatorname{Im} \varphi_{j}$. We want to prove that $K$ is a direct summand of $M$. Let $i_{1}: M_{1} \rightarrow M, i_{2}: M_{2} \rightarrow M$ be the canonical inclusions and let $\pi_{2}: M \rightarrow M_{2}$ be the canonical projection. Let $j \in J$. Since $M_{2}$ is dual Baer, $\operatorname{Im}\left(\pi_{2} \varphi_{j} i_{2}\right)=\pi_{2}\left(\varphi_{j}\left(M_{2}\right)\right)$ is a direct summand of $M_{2}$. Since $M_{2}$ is relative $d$ to $M_{1}$, we have $\operatorname{Im}\left(\pi_{2} \varphi_{j} i_{1}\right)=\pi_{2}\left(\varphi_{j}\left(M_{1}\right)\right)$ is a direct summand of $M_{2}$. As $M_{2}$ has SSSP, $\pi_{2}\left(\operatorname{Im} \varphi_{j}\right)=\pi_{2}\left(\varphi_{j}\left(M_{2}\right)\right)+\pi_{2}\left(\varphi_{j}\left(M_{1}\right)\right)$ is a direct summand of $M_{2}$. Hence $\pi_{2}(K)$ is a direct summand of $M_{2}$ since $M_{2}$ has SSSP. It follows that $\pi_{2}(K)+M_{1}$ is a direct summand of $M$. But it is clear that $\pi_{2}(K)+M_{1}=K+M_{1}$. Thus $K+M_{1}$ is a direct summand of $M$. Let $L$ and $E$ be two submodules of $M$ such that $M=\left(K+M_{1}\right) \oplus L$ and $K+M_{1}=E \oplus M_{1}$. Then $M=E \oplus L \oplus M_{1}$. Thus $M_{2} \cong E \oplus L$. Since $M_{2}$ is $M_{1^{-}}$ projective, $E$ is $M_{1}$-projective. So there exists a submodule $K^{\prime} \leq K$ such that $K^{\prime} \oplus$ $M_{1}=K+M_{1}$ (see [2, 4.12]). Thus $M=K^{\prime} \oplus M_{1} \oplus L$. Hence $K=K^{\prime} \oplus\left[\left(M_{1} \oplus L\right) \cap\right.$ $K]=K^{\prime} \oplus\left(M_{1} \cap K\right)$. Now we consider the homomorphism $\pi \varphi_{j}: M \rightarrow M_{1}$ where $\pi$ : $M=K^{\prime} \oplus M_{1} \oplus L \rightarrow M_{1}$ is the canonical projection. Since $M_{1}$ is dual Baer and $M_{2}$ is relative $d$ to $M_{1}, \operatorname{Im}\left(\pi \varphi_{j}\right)=\pi\left(\varphi_{j}\left(M_{1}\right)\right)+\pi\left(\varphi_{j}\left(M_{2}\right)\right)$ is a direct summand of $M_{1}$ (Theorem 2.1). Hence $\sum_{j \in J} \operatorname{Im}\left(\pi \varphi_{j}\right)$ is a direct summand of $M_{1}$ because $M_{1}$ has SSSP. But $\sum_{j \in J} \operatorname{Im}\left(\pi \varphi_{j}\right)=\pi\left(\sum_{j \in J} \operatorname{Im} \varphi_{j}\right)=\pi(K)=M_{1} \cap K$. Then $M_{1} \cap K$ is a direct summand of $M_{1}$. Therefore $K=K^{\prime} \oplus\left(M_{1} \cap K\right)$ is a direct summand of $M$.

Lemma 3.9. Let $M_{1}, M_{2}$ and $M_{3}$ be dual Baer relatively d-modules. Assume that $M_{2}$ is $M_{1}$-projective (or $M_{1}$ is $M_{2}$-projective). Then $M_{1} \oplus M_{2}$ and $M_{3}$ are relatively d-modules.

Proof. Let $\varphi: M_{1} \oplus M_{2} \longrightarrow M_{3}$ be any homomorphism. Then $\operatorname{Im}\left(\varphi i_{1}\right)=\varphi\left(M_{1}\right)$ and $\operatorname{Im}\left(\varphi i_{2}\right)=\varphi\left(M_{2}\right)$ are direct summands of $M_{3}$, where $i_{1}: M_{1} \rightarrow M_{1} \oplus M_{2}$ and $i_{2}: M_{2} \rightarrow M_{1} \oplus M_{2}$ are the canonical inclusions $\left(M_{1}\right.$ and $M_{2}$ are relative $d$ to $\left.M_{3}\right)$. Since $M_{3}$ has $\operatorname{SSSP}, \operatorname{Im} \varphi$ is a direct summand of $M_{3}$. Now let $\psi: M_{3} \longrightarrow M_{1} \oplus M_{2}$ be any homomorphism. Since $M_{3}$ is relative $d$ to $M_{2}, \operatorname{Im}\left(\pi_{2} \psi\right)$ is a direct summand of $M_{2}$, where $\pi_{2}: M_{1} \oplus M_{2} \rightarrow M_{2}$ is the canonical projection. Therefore $\pi_{2}(\operatorname{Im} \psi)$ is a direct summand of $M_{2}$. Thus $\pi_{2}(\operatorname{Im} \psi)+M_{1}$ is a direct summand of $M_{1} \oplus M_{2}$. 
But $\pi_{2}(\operatorname{Im} \psi)+M_{1}=\operatorname{Im} \psi+M_{1}$. Then $\operatorname{Im} \psi+M_{1}$ is a direct summand of $M_{1} \oplus M_{2}$. Let $L$ and $E$ be submodules of $M_{1} \oplus M_{2}$ such that $\left(\operatorname{Im} \psi+M_{1}\right) \oplus L=M_{1} \oplus M_{2}$ and $\operatorname{Im} \psi+M_{1}=E \oplus M_{1}$. Thus $E \oplus L \cong M_{2}$. Since $M_{2}$ is $M_{1}$-projective, $E$ is $M_{1}$-projective. So there exists $F \leq \operatorname{Im} \psi$ such that $F \oplus M_{1}=\operatorname{Im} \psi+M_{1}$ (see [2, 4.12]). Hence $F \oplus M_{1} \oplus L=M_{1} \oplus M_{2}$. Therefore $\operatorname{Im} \psi=F \oplus\left[\operatorname{Im} \psi \cap\left(M_{1} \oplus L\right)\right]=$ $F \oplus\left(\operatorname{Im} \psi \cap M_{1}\right)$. Let $\pi: F \oplus M_{1} \oplus L \rightarrow M_{1}$ be the canonical projection. Consider the homomorphism $\pi \psi: M_{3} \rightarrow M_{1}$. We have $\operatorname{Im}(\pi \psi)=\pi(\operatorname{Im} \psi)=\pi(F \oplus[\operatorname{Im} \psi \cap$ $\left.\left.M_{1}\right]\right)=\operatorname{Im} \psi \cap M_{1}$. Since $M_{3}$ is relative $d$ to $M_{1}, \operatorname{Im} \psi \cap M_{1}$ is a direct summand of $M_{1}$. Therefore $\operatorname{Im} \psi$ is a direct summand of $M_{1} \oplus M_{2}$, and the proof is complete.

TheOrem 3.10. Let $M_{1}, \ldots, M_{n}$ be dual Baer modules, where $n \in \mathbb{N}$. Assume that, for any $i \neq j, M_{i}$ and $M_{j}$ are relatively d-modules and for any $i<j, M_{i}$ is $M_{j}$-projective. Then $M=\oplus_{i=1}^{n} M_{i}$ is dual Baer.

Proof. By Lemmas 3.8 and 3.9.

ACKNOWLEDGEMENTS. This work has been done during a visit of the second author to the Department of Mathematics, Hacettepe University, and he wishes to thank the first author and her family for the invitation and their hospitality. The authors express their sincere thanks to the referee for the valuable suggestions and comments.

\section{REFERENCES}

1. R. Camps and W. Dicks, On semi-local rings, Israel J. Math. 81 (1993), 203-211.

2. J. Clark, C. Lomp, N. Vanaja and R. Wisbauer, Lifting modules, in Supplements and Projectivity in Module Theory Series: Frontiers in Mathematics (Birkhauser Verlag, Basel-Boston-Berlin, 2006).

3. A. Facchini, Module theory: Endomorphism rings and direct sum decompositions in some classes of modules (Birkhauser, Basel-Boston-Berlin, 1998).

4. L. Ganesan and N. Vanaja, Modules for which every submodule has a unique coclosure, Commun. Algebra 30(5) (2002), 2355-2377. 73-92.

5. J. L. Garcia, Properties of direct summands of modules, Commun. Algebra 17(1) (1989),

6. A. Idelhadj and R. Tribak, Modules for which every submodule has a supplement that is a direct summand, Arab. J. Sci. Eng. Sect. C Theme Issues 25(2) (2000), 179-189.

7. I. Kaplansky, Modules over Dedekind rings and valuation rings, Trans. Amer. Math. Soc. 72 (1952), 327-340.

8. F. Kasch, Modules and rings (Academic Press, New York, 1982).

9. T. Y. Lam, Lectures on modules and rings (Springer-Verlag, New York, 1999).

10. S. H. Mohamed and B. J. Müller, Continuous and discrete modules (London Math. Soc. LNS 147: Cambridge University Press, England, UK, 1990).

11. S. T. Rizvi and C. S. Roman, Baer and quasi-Baer modules, Commun. Algebra 32(1) (2004), 103-123.

12. S. T. Rizvi and C. S. Roman, On $\mathcal{K}$-nonsingular modules and applications, Commun. Algebra 35 (2007), 1-22.

13. D. W. Sharpe and P. Vamos, Injective modules (Cambridge University Press, England, UK, 1972).

14. Y. Talebi and N. Vanaja, Torsion theory cogenerated by M-small modules, Commun. Algebra 30(3) (2002), 1449-1460.

15. R. Wisbauer, Foundations of module and ring theory (Gordon and Breach, Reading, PA, 1991). 